\title{
Assessment of Breast Volume Change after Transverse Rectus Abdominis Myocutaneous Flap
}

\author{
Sang Uk Park, Jeong Su Shim \\ Department of Plastic and Reconstructive Surgery, Catholic University of Daegu School of Medicine, Daegu, Korea
}

Background The evaluation of a breast after breast reconstruction depends on a surgeon's subjective criteria. We used computed tomography (CT) scans to obtain an objective evaluation of the postoperative results by measuring the breast volume of patients who had undergone breast reconstruction using pedicled transverse rectus abdominis myocutaneous (TRAM) flaps. This research will help in the objective postoperative evaluation of reconstructed breasts, and also in the preoperative flap size designs.

Methods A total of 27 patients underwent breast reconstruction using pedicled TRAM flaps after mastectomy from September 2007 to July 2010. Of these, 10 patients who were followed up and underwent CT scans 2 or more times during the follow-up period were included in this study. We evaluated the change in breast volume over time using CT scans, and the interval breast volume change between CT scans.

Results All of the 10 patients' reconstructed breasts showed a volume decrease over time. The breast volume changes in the intervals between CT scans were as follows: 5.65\% decrease between the first CT and second CT scan, 2.3\% decrease between the second CT and third CT scan, (statistically significant) and 1.89\% decrease between the third CT and forth CT scan. (not statistically significant).

Conclusions This research shows the possibility of objectively evaluating the postoperative breast volume changes. The findings will be helpful in designing the size of TRAM flaps to use on defects after mastectomy. Based on these results, we should also closely observe the reconstructed breast volume for at least 2 years.

Keywords Mammaplasty / Multidetector computed tomography / Organ size
Correspondence: Jeong Su Shim Department of Plastic and Reconstructive Surgery, Catholic University of Daegu School of Medicine, 33 Duryugongwon-ro 17-gil, Nam-gu, Daegu 705-718, Korea

Tel: +82-53-650-4578

Fax: +82-53-650-4584

E-mail: 21csue@hanmail.net

No potential conflict of interest relevant to this article was reported.

\section{INTRODUCTION}

Breast reconstruction with flap surgery has fewer complications compared with breast reconstruction with artificial implants, such as implant displacement, infection, and capsular contracture [1]. This procedure results in a more natural breast than does breast reconstruction with artificial implants [2]. However, changes in breast volume occur over time following breast re- construction with flap surgery [3]. Quantitative measurements of breast volume in follow-up periods after breast reconstruction using a flap have not yet been reported.

Therefore, in the current study, postoperative breast volume changes were measured after breast reconstruction using transverse rectus abdominis myocutaneous (TRAM) flap. The results of these measurements should be helpful for deciding the flap size based on the defect after mastectomy and more objectively 
evaluating the postoperative results.

\section{METHODS}

\section{Subjects}

A total of 27 patients underwent immediate breast reconstructions using TRAM flaps after mastectomy from September 2007 to July 2010. The patients that were examined using positron emission tomography/computed tomography (PET/CT) or magnetic resonance imaging (MRI) were excluded, and the patients followed up using CT were included in the study. The patients who had received radiation therapy after mastectomy were also excluded.

CT scans were routinely performed for surveillance in the general surgery department to detect metastasis and recurrence of cancer. Therefore, CT scans did not need to be performed for our breast measurement study.

\section{The method of breast volume measurement}

Consecutive CT scans were performed at $1 \mathrm{~mm}$ intervals, $1 \mathrm{~mm}$ thickness at $120 \mathrm{kV}$, and $170 \mathrm{mAs}$ with a v64 channel multidetector computed tomography (MDCT) device (LightSpeed,
GE Healthcare, Milwaukee, WI, USA) while the patients were lying supine, holding their breaths, and raising both hands over their heads. The breast volumes were examined using the Infinitt program (Infinitt Health Care, Seoul, Korea). We checked each section of the breast such as the section visible in a CT axial view, and changed each section into a three-dimensional image and measured the volume. The same surgeon checked the volume 3 times for each patient, and the median value was set as the breast volume.

When we measured the breast volume, we set the range of the breast from the posterior margin, anterior of the pectoralis major to the anterior margin, skin, and nipple-areola complex. On the axial view, we set the range of the breast from the starting part of the breast crest to the ending part of the breast crest. The medial side of the breast was the lateral border of the sternum, and the lateral side of the breast was the mid-axillary line (Fig. 1).

\section{The method of evaluation}

We compared the changes in breast volume over time. In all 10 patients, the first CT scan was performed at about 9 months after breast reconstruction (average, 9.2 months), and the in-

\section{Fig. 1. Author's method for measuring the breast volume}

(A) The boundary of the breast is selected in each axial view section of computed tomography. (B) The anterior view of a 3-dimensional (3D) reconstructed image. (C) The basal view of a 3D reconstructed image. (D) The breast volume is measured.
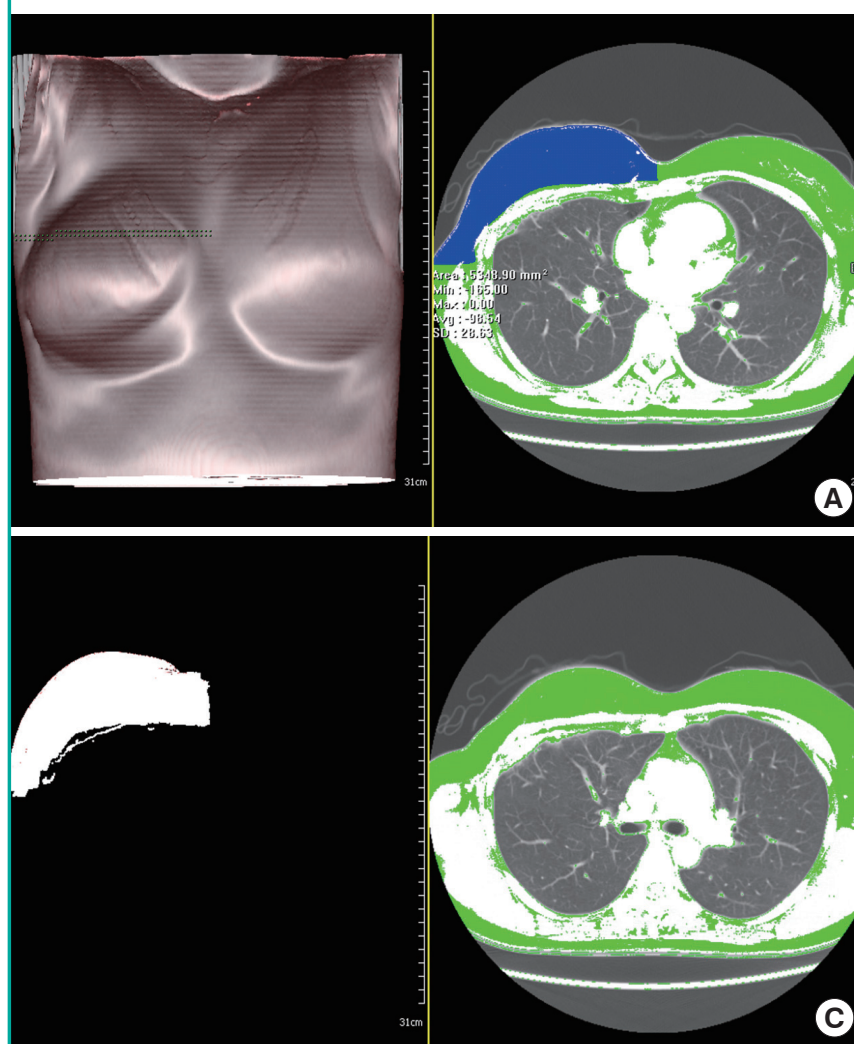
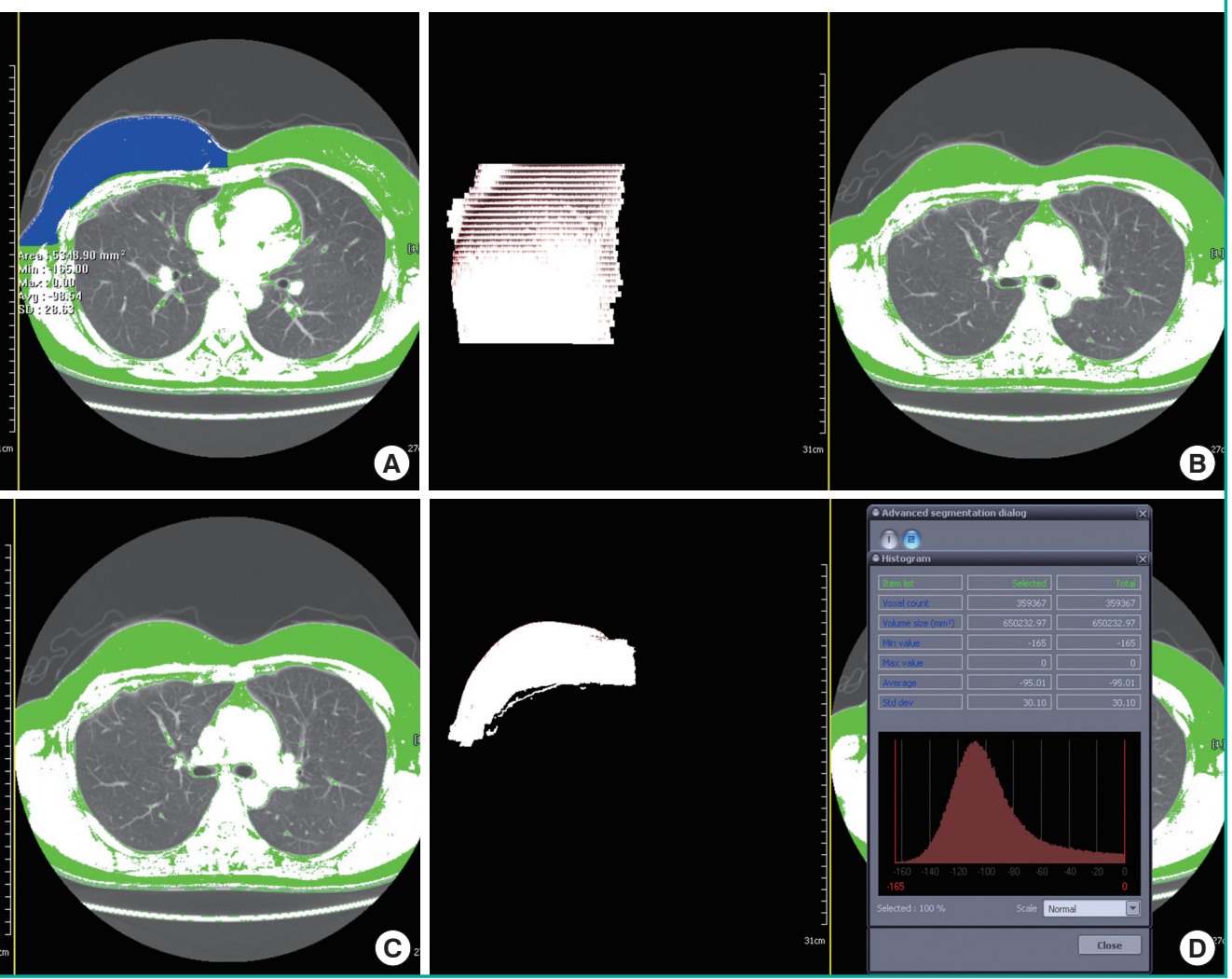
terval period between CT scans was about 1 year (average, 11.7 months).

We evaluated the breast volume change between each CT scan. We defined the interval volume change (VC) between the first CT and second CT scan as "VC1" $(\mathrm{n}=10)$, the volume change between the second CT and third CT as "VC2" $(n=7)$, and the volume change between third $\mathrm{CT}$ and fourth $\mathrm{CT}$ as "VC3" (n=4) (Fig. 2).

We evaluated the mean change of VC and VC in proportion to the breast volume in the earlier CT. We used the Wilcoxon signed-rank test for statistical verification.

\section{RESULTS}

All 10 patients underwent modified radical mastectomy in the general surgery department of our hospital. All of the patients underwent pedicled TRAM flap using the contralateral superior epigastric artery. All 10 patients were diagnosed with invasive ductal carcinoma. The patients were categorized according to stage as follows: stage I, 2 patients; stage IIa, 7 patients; and stage IIIa, 1 patient. All 10 patients were treated with the same regimen of chemotherapy and hormone therapy (Table 1). The mean age of the patients was 44.3 years, and the mean follow-up period was 3.8 years.

\section{Volume changes of breasts with lesions treated with TRAM flap}

Of the 10 patients, the volume of the breast that had the lesion decreased on average over time (Table 2): VC1 was 5.65\%, VC2 was $2.3 \%$, and $\mathrm{VC} 3$ was $1.89 \%$, decreased in proportion to the earlier CT. The differences in VC1 (P-value, 0.005) and VC2

\section{Fig. 2. This figure shows what we evaluated and what is VC}

We defined "volume change (VC)" as interval volume change between computed tomography (CT) scans. In this study, VC was breast volume change proportional to breast volume in the former CT scan. TRAM, transverse rectus abdominis myocutaneous.

\begin{tabular}{|c|c|c|c|c|c|c|c|c|}
\hline & & 1st CT & VC1 & 2nd CT & VC2 & 3rd CT & VC3 & 4th CT \\
\hline $\begin{array}{l}\text { Patient } 1 \\
\text { Patient } 2\end{array}$ & $\begin{array}{c}\text { Breast } \\
\text { reconstruction } \\
\text { using TRAM }\end{array}$ & & & & & & & \\
\hline Patient 3 & & & & & & & & \\
\hline Patient 4 & & & & & & & & \\
\hline Patient 5 & & & & & & & & \\
\hline Patient 6 & & & & & & & & \\
\hline Patient 7 & & & & & & & & \\
\hline Patient 8 & & & & & & & & \\
\hline Patient 9 & & & & & & & & \\
\hline Patient 10 & & & & & & & & \\
\hline
\end{tabular}

(P-value, 0.018) were statistically significant. However, the difference in VC3 was not statistically significant (P-value, 0.068) (Figs. 3, 4)

\section{DISCUSSION}

The goal of breast reconstruction is for the breast that had the lesion to resemble the preoperative breast. For this, symmetry of both breasts needs to be satisfied. During the postoperative follow-up period, the evaluation criteria of the changes in the breast and symmetry of both breasts depends almost entirely on the surgeon's subjective criteria.

The measurement of breast volume is clearly necessary when performing breast reconstruction and mammoplasty. Using this measurement, we determined the size of the breast implant and the increase of the patient's postoperative satisfaction. Many methods are available to measure breast volume, but no standard method has yet been established; therefore, at our hospital,

\section{Table 1 . Clinical information of the study group}

\begin{tabular}{|c|c|c|c|c|c|}
\hline Patient & Pathological finding & Clinical stage & CTx & HTx & RTx \\
\hline 1 & IDC & Ila & 0 & 0 & $x$ \\
\hline 2 & IDC & lla & 0 & 0 & $x$ \\
\hline 3 & IDC & lla & 0 & 0 & $x$ \\
\hline 4 & IDC & Ila & 0 & 0 & $x$ \\
\hline 5 & IDC & \|la & 0 & 0 & $x$ \\
\hline 6 & IDC & Ila & 0 & 0 & $x$ \\
\hline 7 & IDC & Ila & 0 & 0 & $x$ \\
\hline 8 & IDC & I & 0 & 0 & $x$ \\
\hline 9 & IDC & Ila & 0 & 0 & $x$ \\
\hline 10 & IDC & I & 0 & 0 & $x$ \\
\hline
\end{tabular}

Table 2. Volumetric changes of the reconstructed breasts using TRAM flap

\begin{tabular}{|lcccc|}
\hline \multicolumn{5}{|c|}{$\begin{array}{c}\text { Measured breast volume in } \\
\text { the operated side (mL) }\end{array}$} \\
\cline { 2 - 5 } Patients & $\begin{array}{c}\text { 1st CT } \\
\text { after OP }\end{array}$ & $\begin{array}{c}\text { 2nd CT } \\
\text { after OP }\end{array}$ & $\begin{array}{c}\text { 3rd CT } \\
\text { after OP }\end{array}$ & $\begin{array}{c}\text { 4th CT } \\
\text { after OP }\end{array}$ \\
\hline Patient 1 & $847 \pm 13$ & $802 \pm 9$ \\
Patient 2 & $543 \pm 4$ & $514 \pm 12$ & \\
Patient 3 & $471 \pm 10$ & $442 \pm 15$ & $432 \pm 6$ & \\
Patient 4 & $410 \pm 10$ & $409 \pm 5$ & $409 \pm 6$ & \\
Patient 5 & $309 \pm 12$ & $299 \pm 7$ & $298 \pm 5$ & \\
Patient 6 & $238 \pm 5$ & $228 \pm 6$ & $223 \pm 11$ & \\
Patient 7 & $511 \pm 9$ & $471 \pm 8$ & $451 \pm 3$ & $439 \pm 4$ \\
Patient 8 & $783 \pm 4$ & $742 \pm 6$ & $721 \pm 7$ & $710 \pm 2$ \\
Patient 9 & $1101 \pm 9$ & $1029 \pm 6$ & $992 \pm 8$ & $986 \pm 4$ \\
Patient 10 & $932 \pm 3$ & $862 \pm 7$ & $853 \pm 4$ & $825 \pm 11$ \\
\hline Values are presented as mean \pm standard deviation. \\
TRAM, transverse rectus abdominis myocutaneous; CT, computed tomography; \\
OP, operation. \\
\hline 5 \\
\hline
\end{tabular}




\section{Fig. 3. Volume change of the reconstructed breast using TRAM flap in patient 7}

(A) Measurement value of the breast on the first CT. (B) Measurement value of the breast in the fourth CT, 3 years and 4 months later. This figure shows the volumetric decrease of the breast over time. TRAM, transverse rectus abdominis myocutaneous; CT, computed tomography.

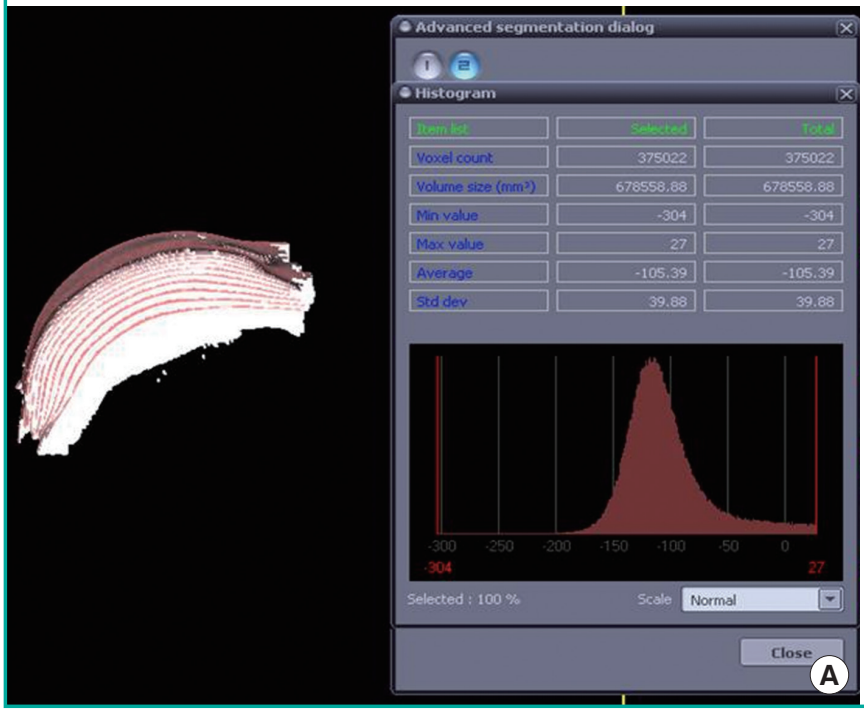

Fig. 4. This figure shows that breast volume changed in the interval between CT scans

The first (5.65\%) and second (2.3\%) breast volume changes are statistically significant. However, the third breast volume change $(1.89 \%)$ is not statistically significant. These results mean that the reconstructed breast volume change after 3 years can be ignored; therefore, the volume of a reconstructed breast should be closely observed for at least 2 years. TRAM, transverse rectus abdominis myocutaneous; CT, computed tomography.

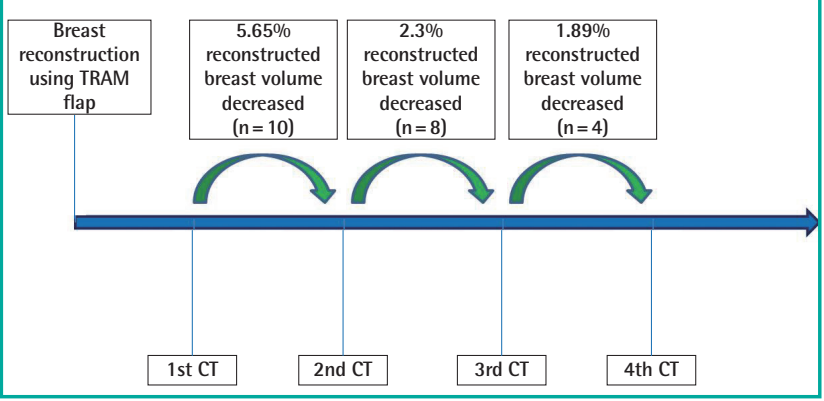

we depend on the surgeon's subjective decision when making preoperative plans or postoperative predictions.

In a prior study, Tegrmeier [4] measured breast volume using overflowing water when the breast was dipped in a bowl filled with water. Grossman and Roudner [5] measured the breast volume using a self-constructed device. Edsander-Nord et al. [6] measured the volume using a thermoplastic casting. Methods that measure breast volume changes using three-dimensional body surface images from devices such as laser scanners [7] and stereo photographs [8] have also been introduced. Recently, with the development of radiologic technology, indirect breast volume measurements using MRI and its usefulness have been
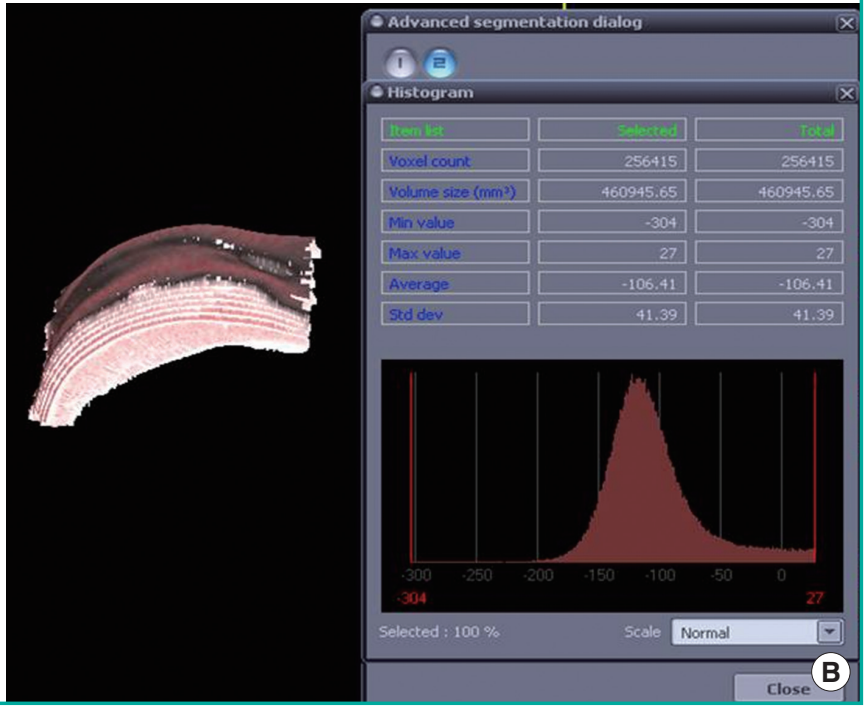

reported $[9,10]$.

The method for measuring breast volume with an imaging device involves less discomfort for patients, and we can ignore the changes caused by the patient's position. CT is less expensive and more cost-effective than MRI. CT has been long used for follow-up evaluations on the recurrence of breast cancer after breast resection and metastasis of localized lymph node.

The measurement of volume using a CT scan was introduced previously in the diagnosis of a blow-out fracture. Lee et al. [11] argued that the exact diagnosis and prediction of volume change in an injured orbit over time could be obtained through a CT scan. The prediction of orbital volume change through the followup period is important for determining whether surgery should be performed or not. Similarly, in this study, objective and quantitative data could be obtained through CT scans, and the exact preoperative plan and postoperative change of the breast were predicted.

To satisfy the symmetry of both breasts following breast reconstruction, Stevenson and Goldstein [12] increased patient satisfaction by performing mastopexy and breast reduction on the contralateral breast, but this was performed on a Western women who had large breasts and on other patients who had ptosis of the breast. Thus, applying this to East Asian women who tend to have small to medium-sized breasts is not appropriate. Therefore, an accurate size of the flap inserted into the breast with the lesion has to be considered for breast reconstruction.

Amir et al. [13] reported that proper flap size was obtained by measuring the resected breast tissue and the flap volume using Archimedes' rule for breast reconstruction with a free TRAM 
flap. Lazarus and Hudson [14] also reported that the proper flap size was obtained by measuring the resected breast tissue and flaps using scales. However, after breast reconstruction, the volume of the breast that has been operated on began to change. Amir et al. [13] determined the proper volume of the flap, which was calculated from the actual volume of the flap, plus an additional $10 \%$ of its volume. However, no reports have mentioned volume changes that occur over time. Therefore, this study aimed to determine the VC of flaps over time by measuring the $\mathrm{VC}$ in the operated breast using CT scanning. We found that after more than 3 years of follow-up, the change in breast volume could be ignored. Therefore, we should closely observe the breast volume for 2 years.

Based on these results, surgeons and patients were more satisfied with the results obtained by applying an over-correction of the flap size when the design of the flap was made.

In this study, inter-observer bias was minimized because only one examiner measured all of the breast volumes. Intra-observer bias was also minimized by using a mean value of three separate measurements.

Measuring breast volume using CT scans also depends on the examiner's subjective judgment, and the inaccuracy of measurements can cause apparent differences between breast volumes. This method considered only the volume of breasts in evaluating symmetry and did not consider the shapes of the breasts.

This study has some limitations. Because only a few cases were examined, the results cannot be broadly generalized. Moreover, the follow-up periods were short and the radiation effect on the flap was ignored; there was also the risk of unnecessary radiation exposure. Therefore, this study's findings are limited to generalization to the following types of patients: patients who want immediate breast reconstruction after mastectomy, patients who have a high risk potential for local or distant metastasis, which should be observed using CT scanning during the follow-up period, and patients who do not need adjuvant radiation therapy after breast reconstruction.

However, measuring the volume of the breast using a CT scan is cost-effective and does not cause patient discomfort. Therefore, this method is an objective and effective method for measuring breast volume and can be very helpful in designing the size of TRAM flaps on the defect after mastectomy.

\section{REFERENCES}

1. Levine SM, Lester ME, Fontenot B, et al. Perforator flap breast reconstruction after unsatisfactory implant reconstruction. Ann Plast Surg 2011;66:513-7.

2. Vega SJ, Bossert RP, Serletti JM. Improving outcomes in bilateral breast reconstruction using autogenous tissue. Ann Plast Surg 2006;56:487-90.

3. Mandrekas AD, Zambacos GJ, Zervoudis S. Tram flap breast reconstruction and weight fluctuations: it is alive! Plast Reconstr Surg 2003;112:696-7.

4. Tegrmeier RE. A convenient, effective mammary sizer. Aesth Plast Surg 1979;3.

5. Grossman AJ, Roudner LA. A simple means for accurate breast volume determination. Plast Reconstr Surg 1980; 66:851-2.

6. Edsander-Nord A, Wickman M, Jurell G. Measurement of breast volume with thermoplastic casts. Scand J Plast Reconstr Surg Hand Surg 1996;30:129-32.

7. Galdino GM, Nahabedian M, Chiaramonte M, et al. Clinical applications of three-dimensional photography in breast surgery. Plast Reconstr Surg 2002;110:58-70.

8. Kovacs L, Eder M, Hollweck R, et al. Comparison between breast volume measurement using $3 \mathrm{D}$ surface imaging and classical techniques. Breast 2007;16:137-45.

9. Herold C, Reichelt A, Stieglitz LH, et al. MRI-based breast volumetry-evaluation of three different software solutions. J Digit Imaging 2010;23:603-10.

10. Caruso MK, Guillot TS, Nguyen T, et al. The cost effectiveness of three different measures of breast volume. Aesthetic Plast Surg 2006;30:16-20.

11. Lee JK, Kweon YD, Kim CS, et al. Orbital volume change measured by computed tomography in blow out fracture. J Korean Soc Plast Reconstr Surg 2000;27:437-43.

12. Stevenson TR, Goldstein JA. TRAM flap breast reconstruction and contralateral reduction or mastopexy. Plast Reconstr Surg 1993;92:228-33.

13. Amir A, Silfen R, Hauben DJ. Use of Archimedes' law for measuring the volume of the TRAM flap in immediate breast reconstruction. Plast Reconstr Surg 1999;103:1329.

14. Lazarus D, Hudson DA. A simple method for determining the weight of the TRAM flap intraoperatively at the time of breast reconstruction. Plast Reconstr Surg 2001;107:818-22. 


\section{Discussion}

\section{Assessment of Breast Volume Change after Transverse Rectus Abdominis Myocutaneous Flap}

\section{Jun Sik Kim}

Department of Plastic and Reconstructive Surgery, Gyeongsang National University Hospital, Gyeongsang National University School of Medicine, Jinju, Korea

It is well known that breast reconstruction after breast cancer extirpation plays an important role in offering benefits to restore symmetry, correct body image, and improve psychosocial wellbeing. Patients' desire for the restoration of body image after breast cancer extirpation is increasing markedly. There are a variety of reconstructive options available to patients. There has been a great deal of debate over the advantages of reconstructive surgical methods of the breast, but almost all plastic surgeons agree that breast reconstruction with autologous tissue is the most suitable surgical option for breast cancer patients. However, as the author observed, change in breast volume occurs over time, following breast reconstruction with autologous tissue.

Amir et al. [1] determined the volume needed for an inserted flap, figured from the actual volume of the removed flap plus an additional 10 percent of its volume, given a predictable volume decrease. However, while this quantitative calculation of additional volume needed for an inserted flap is useful for flap shaping and volume measurement, it is not adequate for measurement of the reconstructed breast volume during follow-up.

Let it first be said that I agree with the author's decision to measure the postoperative breast volume change using computed tomography (CT) after breast reconstruction with the transverse abdominal myocutaneous island flap. It is a novel method for measurement of breast volume change over time during followup.

However, there are some limitations to this study. First, for the consecutive $\mathrm{CT}$ scanning, the patient was laid in the supine position while holding postoperative tissue edema, the position of the inserted flap, the status of the breast skin envelopes, and the ratio of the volume of the inserted flap versus the original breast skin her breath and raising both hands over her head. Normal breasts of women sag laterally in this position, but a reconstructed breast of a patient does not initially sag because of envelope. All of these factors could have introduced a measurement bias by repetition of CT scanning through uncorrectable flap motion although the author set the fixed bony point at the medial and lateral border. After the author checked each section of the axial CT scan, the sections were transformed into three-dimensional (3D) images. The process of this transformation would also introduce a bias in each axial scanBreast volume measurement using a 3D laser surface image change rather than in a CT scan is more accurate. Furthermore, a 3D surface image obtained using non-invasive recording in the standing position provides the ability to quantitatively evaluate symmetry, volume, shape, contour, and surface, and also measure distance [2]. Second, this study included a very small number of patients. The author classified the patients into 4 groups, and each group was composed of 2 or 3 patients. This means that the volume change in one patient from the same group differs as much as the mean value of the group. To reduce statistical bias, each group should have had more than 7 patients. Finally, there were cost-benefit effects. The author affirmed that measuring the volume of the breast using a CT scan is cost-effective and does not cause discomfort to the patient. I disagree with the author's point of view on that matter. A 1-mm-thick slice in a follow-up CT scan for breast volume measurement is a much thinner section than routine breast CT scan, which is $5 \mathrm{~mm}$ in thickness in each section. This follow-up CT scan is not covered by insurance in Korea, and it is an additional economic burden to patients.

In conclusion, this article was the first trial for measurement of volume changes in breast reconstruction patients using CT scans. It would have been better if the volume measurement using a CT scan could represent the appearance and symmetry of the breast.

\section{REFERENCES}

1. Amir A, Silfen R, Hauben DJ. Use of Archimedes' law for measuring the volume of the TRAM flap in immediate breast reconstruction. Plast Reconstr Surg 1999;103:1329.

2. Kovacs L, Eder M, Hollweck R, et al. Comparison between breast volume measurement using $3 \mathrm{D}$ surface imaging and classical techniques. Breast 2007;16:137-45.

\section{Correspondence: Jun Sik Kim}

Department of Plastic and Reconstructive Surgery, Gyeongsang National University Hospital,

Gyeongsang National University School of Medicine, 79 Gangnam-ro, Jinju 660-702, Korea

Tel: +82-55-750-8131, Fax: +82-55-758-6240, E-mail: junskim@gshp.gsnu.ac.kr

No potential conflict of interest relevant to this article was reported.

Received: 31 Oct 2012 • Revised: 1 Nov 2012 • Accepted: 2 Nov 2012

pISSN: 2234-6163・ elSSN: 2234-6171

http://dx.doi.org/10.5999/aps.2012.39.6.636 • Arch Plast Surg 2012;39:636 